\title{
Thermodynamics of Efflux Process of Liquids and Gases
}

\author{
E. A. Mikaelian1, Saif A. Mouhammad2* \\ ${ }^{1}$ Gubkin Russian State University of Oil and Gas, Moscow, Russia \\ ${ }^{2}$ Physics Department, Faculty of Science, Taif University, Taif, Kingdom of Saudi Arabia \\ Email: saifnet70@hotmail.com
}

Received 29 March 2015; accepted 11 May 2015; published 14 May 2015

Copyright (C) 2015 by authors and Scientific Research Publishing Inc.

This work is licensed under the Creative Commons Attribution International License (CC BY). http://creativecommons.org/licenses/by/4.0/

(c) (i) Open Access

\begin{abstract}
The main objective of this work is to obtain the calculated ratio of efflux processes for liquids, vapors, gases on the basis of the developed mathematical model, which allows to determine the characteristics of the channel profiles nozzles and diffusers, to solve a number of subsequent applications for analysis modes. On the basis of the calculated ratios are equations of the first law of thermodynamics for the flow of liquids and gases. The obtained calculated ratios are extended for the case of the efflux of compressible liquids, vapors and gases and as a special case, for incompressible liquids. The characteristics of the critical efflux regime liquids, which allows to determine the linear and the mass efflux rate of the critical regime and the calculated characteristics of the channel profiles nozzles and diffusers, Laval nozzles for different modes of operation are obtained.
\end{abstract}

\section{Keywords}

Thermodynamics, Efflux, Compressible, Incompressible, Liquids, Diffusers, Nozzles

\section{Introduction}

The efflux processes are quite common in various technological processes performed with the power technology equipment in the gas and oil industry, in heat engines, pumps, compressor machines, mas-and-heat exchange units, pipelines, in separate elements of machines and devices: nozzles, diffusers, convergent nozzles, mud guns, fittings, locking devices, gate valves, valves, various calibration holes etc. It is worth emphasising a special role in studying the processes of the gas and liquid efflux through various sorts of leakiness and gap [1] [2].

The efflux process can be considered as a special case of the occurrence and distribution of potential work. Effective work in the efflux process is distributed on the work, directly transmitted to the bodies of external sys-

Corresponding author. 
tem (in our case in the efflux process this work is absent: $\delta W_{c z}^{*}=0$ ) and to a change in the energy of external position of working medium itself $\left(d e_{c z}\right)$. The last term, in turn, consists of the kinetic energy $d\left(c^{2} / 2\right)$ and the potential energy $(g d z)$.

Thus, the initial equation of the theoretical efflux process has the following form:

$$
\delta W=-V d P=d\left(c^{2} / 2\right)+g d z .
$$

Switching to the real efflux processes then is carried out by introducing correction factors: velocity rates $(\varphi)$ and flow rates $(\phi)$. The integral of the initial equation of efflux for the expression of potential flow work from the initial 1 to the final section 2 of a flow has the form as follows:

$$
\begin{aligned}
& W_{12}=c_{2}^{2} / 2-c_{1}^{2} / 2+g\left(z_{2}-z_{1}\right) ; \\
& W_{12}=\left[1-\left(P_{2} / P_{1}\right)^{(n-1) / n}\right] P_{1} V_{1} n /(n-1) .
\end{aligned}
$$

The rate of gas efflux in the initial section can be considered as a result of the efflux of a conditional initial state 0 - 0 at zero velocity $c_{0}=0$; with the graded level $z_{0}=z_{2}$ and pressure $P_{0}$.

Then the calculated expression for the potential work and linear velocity of the efflux of the final section of the flow is determined by the following equations:

$$
\begin{aligned}
& W_{02}=W_{12}+W_{01}=\left[1-\left(P_{2} / P_{0}\right)^{(n-1) / n}\right] P_{0} V_{0} n /(n-1) ; \\
& C_{2}=\left(2 W_{02}\right)^{0.5}=\left[2 W_{12}+c_{1}^{2}+2 g\left(z_{1}+z_{2}\right)\right]^{0.5}
\end{aligned}
$$

The theoretical efflux process is regarded as an adiabatic one, then based on the first law of thermodynamics for the flow the potential flow work is determined as the specific heat drop of the flow equal to the difference between the heat content (enthalpy) of it, taken with the opposite sign [3] [4]:

$$
W_{12}=h_{1}-h_{2} ; \quad q_{12}=0 .
$$

Further the mass rate of the efflux is entered in calculations:

$$
u=G / f=V \rho / f=\rho c,
$$

where $f$ - the cross section of the flow under consideration; $G$ and $V$ are the mass flow rate and volumetric flow rate; $\rho$-liquid density; $c$ - the linear velocity of the liquid in the direction of movement (the average velocity in the section $\mathrm{f}$ in the direction of a normal to this section).

The concept of the mass flow rate in research is most essential. The concept of linear velocity characterises only the kinetic energy of the flow, averaging of such a velocity depends on the flow mode (laminar, transitional, turbulent) and is not identical with the mass flow rate.

The calculated expression of the theoretical mass flow rate at the outlet section is obtained according to the last equation depending on the linear velocity (4) and (5) and the equation of the efflux process:

$$
u_{2}=\left\{\left[1-\left(P_{2} / P_{0}\right)^{(n-1) / n}\right]\left(P_{2} / P_{0}\right)^{2 / n}\left(P_{0} / V_{0}\right) 2 n /(n-1)\right\}^{0.5} .
$$

For transition to the real characteristics of a flow we input correction factors in our calculations:

$$
c=\varphi c_{2} ; \quad u=\phi u_{2} ; \quad G=u f=\phi u_{2} f=\phi c_{2} \rho_{2} f .
$$

In the formula (9) the velocity and flow-rate factors are determined as the ratio of theoretical and actual velocities:

$$
\varphi=c / c_{2}=V / f c_{2} ; \phi=u / u_{2}=G / f u_{2} .
$$

The work of irreversible energy losses associated with the real efflux process:

$$
W^{* *}=\left(c_{2}^{2}-c^{2}\right) / 2=\left(1-\varphi^{2}\right) c_{2}^{2} / 2=\xi c_{2}^{2} / 2
$$


where $\xi$-the factor of energy losses in the real process.

To calculate the velocity and flow rate, as follows from the formulas (10) it is necessary to arrange for mass (volume) measurements of the liquid flow rates [5] [6].

\section{Efflux of Incompressible Liquids}

The initial condition $\left(\rho_{1}=\rho_{2}=\rho=1 / v=\right.$ idem $)$ :

$$
W_{12}=\left(P_{1}-P_{2}\right) / \rho ; W_{02}=\left(P_{0}-P_{2}\right) / \rho .
$$

Further, by using the initial general ratios (5), (7), (9) and Equation (12), we will obtain the calculated ratios for a particular case of the efflux of incompressible liquids:

$$
\begin{gathered}
c_{2}=\left(2 W_{02}\right)^{0.5}=\left[2 W_{12}+c_{1}^{2}+2 g\left(z_{1}+z_{2}\right)\right]^{0.5}=\left[2\left(P_{0}-P_{2}\right) / \rho\right]^{0.5} \\
=\left[2\left(P_{1}-P_{2}\right) / \rho+c_{1}^{2}+2 g\left(z_{1}+z_{2}\right)\right]^{0.5} ; \\
u_{2}=G / f=V \rho / f=\rho c_{2}=\left[2\left(P_{0}-P_{2}\right) \rho\right]^{0.5} ; \\
G=\phi u_{2} f .
\end{gathered}
$$

The obtained ratios can be applied to the efflux of compressible liquids (gases) with the condition of insignificant fluctuations of the densities. In this case, in Formulae (12)-(15) there should be introduced the average density value, for example, as arithmetic mean:

$$
\left(\rho_{1}+\rho_{2}\right) / 2=\rho_{m}
$$

\section{Efflux of Compressible Liquids (Gases)}

The general solution of problems concerning the efflux of compressible liquids is obtained by a corresponding development of the previously obtained initial relationships.

From a consideration of the original ratio (8) it follows that the mass velocity becomes zero for the following values of pressure ratios: 1) $P_{2} / P_{0}=1$, this takes place at the beginning of the efflux $c=0 ; u=c \rho=0$ due to the initial rate; 2) $P_{2} / P_{0}=0$-in the efflux to vacuum at the outlet section $\rho=0 ; u=c \rho=0$ due to density. Within this range, the mass flow rate passes through the maximum (Rolle's theorem). This means the variable factor of the radicand (8) passes through the maximum:

$$
\Psi=\left[1-\left(P_{2} / P_{0}\right)^{(n-1) / n}\right]\left(P_{2} / P_{0}\right)^{2 / n} .
$$

Let us introduce the following designations:

$$
\left(P_{2} / P_{0}\right)=\tau^{n /(n-1)} ;\left(P_{2} / P_{0}\right)^{2 / n}=\tau^{2 /(n-1)},
$$

using the Rolle's theorem for investigating the function to the maximum, we obtain the parameters of the critical mode of efflux for compressible liquids

$$
\begin{gathered}
\tau_{c r}=\left(P_{2} / P_{0}\right)_{c r}^{(n-1) / n}=2 /(n+1), \\
\beta=\left(P_{2} / P_{0}\right)_{c r}=\tau_{c r}^{n /(n-1)}=[2 /(n+1)]^{n /(n-1)}, \\
\Psi_{c r}=\left(1-\tau_{c r}\right) \tau_{c r}^{2 /(n-1)} .
\end{gathered}
$$

Depending on parameters of the critical efflux mode, the linear and mass efflux rates of the critical mode are determined:

$$
c_{c r}=\left[n(P V)_{c r}\right]^{0.5}
$$


Table 1. Characteristic values of the discharge critical mode.

\begin{tabular}{cccccl}
\hline$n$ & 1.1 & 1.2 & 1.3 & 1.4 & \\
\hline$\tau_{c r}=2 /(n+1)$ & 0.953 & 0.909 & 0.870 & 0.833 & \\
$\beta=\tau_{c r}^{n /(n-1)}$ & 0.5847 & 0.5645 & 0.5457 & 0.5283 & $\sim 0.55$ \\
$\Psi_{c r}$ & 1.9677 & 2.0309 & 2.0896 & 2.1443 & $\sim 2.05$ \\
\hline
\end{tabular}

$$
u_{c r}=\left[2 P_{0} \rho_{0} \Psi_{c r} n /(n-1)\right]^{0.5} \text {. }
$$

Table 1 shows the values of the critical discharge characteristics depending on the performance of the efflux process.

\section{Particular Cases of Efflux}

The ideal gas $(P V=R T)$ :

$$
c_{c r}=\left[n R T_{c r}\right]^{0.5} ; \quad u_{c r}=P_{0}\left[2 \Psi_{c r} n /(n-1) R T_{c r}\right]^{0.5},
$$

The incompressible liquids ( $V=$ idem; $n=\infty$ ): $c_{c r}=\infty$.

This means that the critical mode for incompressible fluids is unattainable. The critical linear velocity of the adiabatic efflux $(n=k)$ is the velocity of sound:

$$
a^{*}=\left[k(P V)_{c r}\right]^{0.5}
$$

for the ideal gas:

$$
a^{*}=\left[n R T_{c r}\right]^{0.5}
$$

\section{Conclusion}

According to the energy conservation law, the equation of distribution and occurrence of the potential work of any thermodynamic systems is obtained. Taken as a basis for the theory of the efflux of gases, compressible and incompressible liquids, the characteristic features of the critical mode of the liquid efflux are obtained. The derived calculated ratios will further determine the calculated characteristics of the channel profiles of nozzles and diffusers, Laval nozzles for a range of modes of operation.

\section{References}

[1] Mikaelian, E.A. (2000) Maintenance Energotechnological Equipment, Gas Turbine Gas Compressor Units of Gas Gathering and Transportation. Methodology, Research, Analysis and Practice, Fuel and Energy, Moscow, 304. http://www.dobi.oglib.ru/bgl/5076.html

[2] Mikaelian, E.A. (2001) Improving the Quality, to Ensure Reliability and Safety of the Main Pipelines. In: Margulov, G.D., Ed., Series: Sustainable Energy and Society, Fuel and Energy, Moscow, 640. http://www.dobi.oglib.ru/bgl/4625.html

[3] Vladimirov, A.I. and Kershenbaum, Y.V. (2008) Industrial Safety Compressor Stations. Management of Safety and Reliability. Inter-Sector Foundation "National Institute of Oil and Gas”, Moscow, 640. http://www.mdk-arbat.ru/bookcard?book_id=3304125

[4] Mikaelian, E.A. (2008) Diagnosis Energotechnological Equipment GGPA Based on Various Diagnostic Features. Gas Industry, 4, 59-63.

[5] Mikaelian, E.A. (2014) Determination of the Characteristic Features and Technical Condition of the Gas-Turbine and Gas-Compressor Units of Compressor Stations Based on a Simplified Thermodynamic Model. Quality Management in Oil and Gas Industry, 1, 44-48. http://instoilgas.ru/ukang

[6] Mikaelian, E.A. and Mouhammed, S.A. (2014) Survey Equipment Gas Transmission Systems. Quality Management in Oil and Gas Industry, 4, 29-36. http://instoilgas.ru/ukang 